\title{
Recurrent rhinosporidiosis: a case report
}

\begin{abstract}
Rhinosporidiosis is rare infective chronic granulomatous lesion cause by Rhinosporidium seeberi which occurs universally although endemic in South Asia, notably in Southern India and in Srilanka. The causative agent Rhinosporidium seeberi is intractable to isolation and microbiological culture and shows features of both fungi and protozoa. The great majority of cases occur in upper respiratory sites, notably the anterior nares, nasal cavity- the inferior turbinate's, septum and floor. It is a chronic disease, with frequent recurrence after surgery and occasional dissemination from initial focus .Rhinosporidial lesions in the nasal passages are Polypoidal, granular, red in colour due to pronounced vascularity, with a surface containing yellowish pin head sized spots which represents underlying mature sporangio. The organism is difficult to culture and diagnosis is based on microscopy and histological examination of the lesion. The mainstay of treatment is meticulous complete and wide surgical excision, followed by electro-cautery of the base may ablate recurrence resulting from the spillage of endospore on the adjacent mucosa and recently use of laser is also indicated. We present a thrice operated recurrent case of extensive Rhinosporidiosis involving left nose \& nasopharynx where the disease was removed using bipolar cautery, microdebrider and laser assisted.
\end{abstract}

Volume 10 Issue 6 - 2018

\author{
Inderdeep Singh, Lalrinmawii, Deepika Phogat \\ Department of ENT, INHS Asvini, India
}

Correspondence: Inderdeep Singh, Department of ENT, INHS Asvini, Mumbai-40000I India,Tel +917767834I37,

Email dribsingh@hotmail.com

Received: August 09, 2018 | Published: November 01, 2018

Keywords: rhinosporidiosis, R. seeberi, $\mathrm{CO}_{2}$ laser, electro cautery

\section{Introduction}

The etiological agent of Rhinosporidiosis, R. seeberi, has been a riddle from past 9-10 decades. $R$. seeberi has been variously classified as a prokaryote cyanobacterium of the genus Microcystis, a eukaryote pathogen belonging to the class Mesomycetozoea, and a fungus. ${ }^{1}$ Yet unresolved enigmas in Rhinosporidiosis include the mode of infection mechanisms of spread, mechanisms of immunity, some aspects of histopathology e.g. the significance of trans epidermal elimination of sporangia, the cause of the variation in cell infiltration patterns in Rhinosporidial tissues and their correlations with immune status, and the absence of the Splendore-Hoeppli reaction which is wellmarked in invasive, classical mycoses..$^{2-4}$ It is a chronic disease, with frequent recurrence after surgery, and occasional dissemination from the initial focus which is most commonly seen in upper respiratory sites. It occurs universally, although it is endemic in south Asia, notably southern India and in Sri Lanka. The presumed mode of infection from the natural aquatic habitat of R.seeberi, is through the traumatised epithelium (trans epithelial infection) most commonly in nasal sites. ${ }^{2}$ Trauma from R. seeberi contaminated stones used for mopping-up residual drops of urine is claimed to be responsible for anterior urethral Rhinosporidiosis in the male. ${ }^{2}$ Hypothesized modes of spread, most common being 'Auto-inoculation' responsible for the occurrence of satellite lesions adjacent to granulomas especially in the upper respiratory sites and for local spread, others being haematogenous spread and lymphatic spread. ${ }^{2,3,5}$

\section{Case report}

48 year old male coast guard personnel, a known case of Primary Hypertension and Type II Diabetes Mellitus, presented to this centre with Left nasal mass and bleeding from Left nostril of 04 months duration. There was history of similar complaints for the past 23 years. History of bathing in pond in his village which is in Southern part of India since his childhood. No history of rhinorrhoea /excessive sneezing/trauma to nose. Patient was diagnosed as Rhinosporidiosis and underwent excision of the mass thrice, in 1994, 2006 and 2017. On evaluation, there was a red friable mass filling Left nasal cavity, with spores seen on the surface and bleeds on touch (Figure 1). Computed Tomography of Paranasal sinuses (PNS)-shows Left nasal cavity filled with soft tissue on the anterior aspect, absent middle and inferior turbinate (Figure 2). He underwent Nasal Endoscopic Excision (Microdebrider \& $\mathrm{CO}_{2}$ laser assisted)-mass was removed using Bipolar diathermy and $\mathrm{CO}_{2}$ laser, the lateral wall of the nasal cavity was drilled at basisphenoid, Agger nasi and at medial maxillary wall. $\mathrm{CO}_{2}$ Laser was used at nasal septum and nasopharynx. In our centre we are using $\mathrm{CO}_{2}$ laser especially at the areas of contact over nasal septum nasal floor \& at nasopharynx. Histopathological Examination (HPE) report shows-numerous variably sized thick walled sporangia containing numerous spores, foci of ruptured sporangia also noted with spilling of spores into the adjacent stroma, with chronic inflammatory infiltrates (Figure 3). Post-operative period was uneventful; he was started on Tablet Dapsone $100 \mathrm{mg}, 1$ tab daily for 01 year.

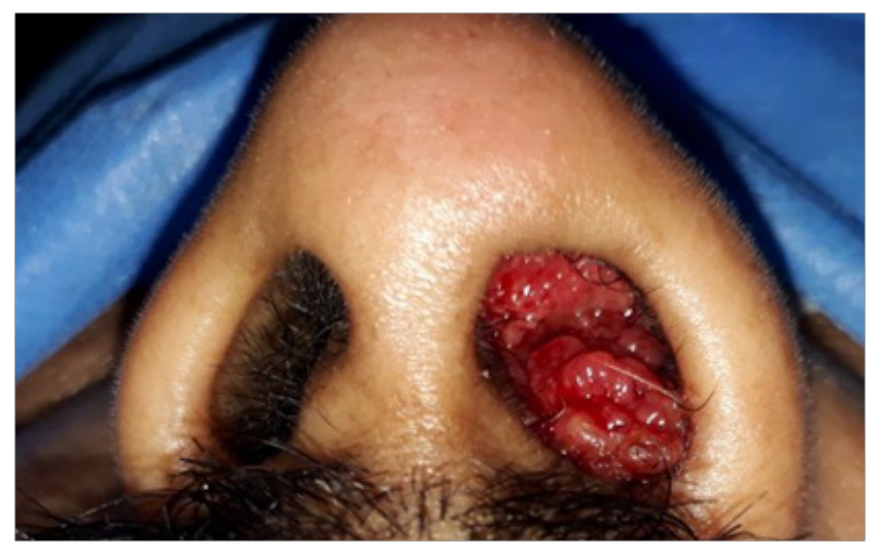

Figure I Mass left nostril. 


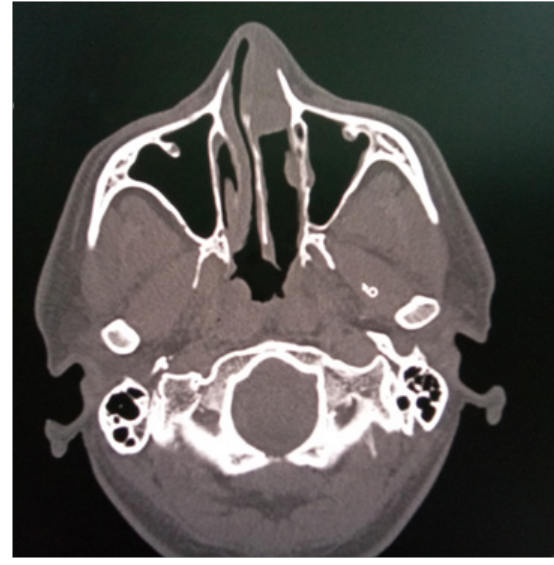

Figure 2 CT PNS showing mass in the Left nasal cavity on the anterior aspect, absent middle and inferior turbinate.

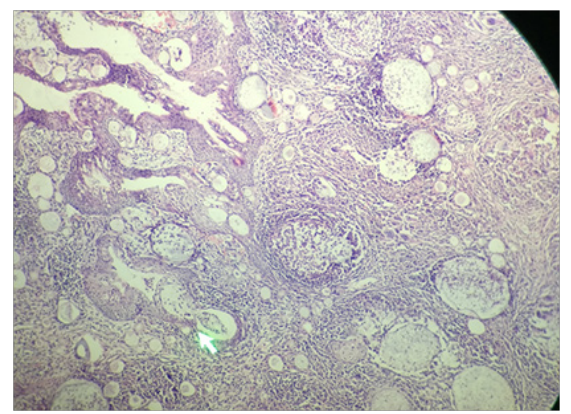

Figure 3 High Power (40X magnifications) Haematoxylin and Eosin staining showing respiratory epithelium, sporangia of different sizes with background of inflammatory infiltrates.

\section{Results}

Our patient had a Recurrent Rhinosporidiosis. He was managed with Nasal Endoscopic Excision (Microdebrider \& $\mathrm{CO}_{2}$ laser assisted). Bulk mass was removed using bipolar diathermy and $\mathrm{CO}_{2}$ laser was used over the surface attachments at nasal septum upto posterior choana and nasopharynx roof to minimise recurrence. The patient was started on Tablet Dapsone 100mg, 1 tab daily for 01 year post operatively to arrest maturation of residual sporangia and accelerate the degenerative changes in them, which will further reduce chances of recurrence. Patient is on regular follow up every 03 monthly and there is no evidence of recurrence after 2 years with well healed nasal cavity as shown in Figure 4. There were no adhesions, synechiae or septal perforation.

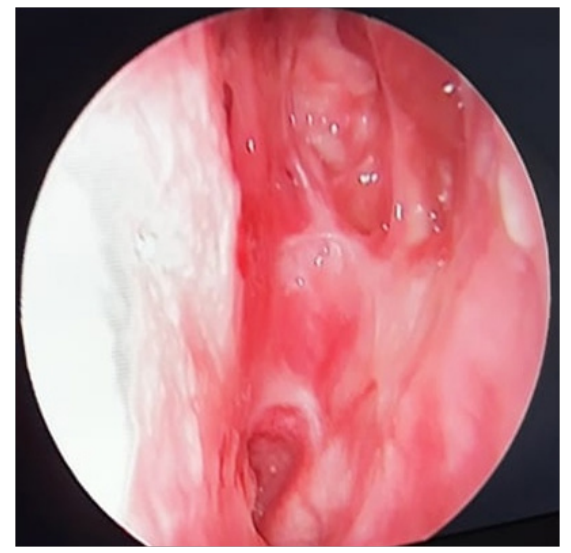

Figure 4 Endoscopic picture of Left nasal cavity after 02 years.

\section{Discussion}

The definitive diagnosis of Rhinosporidiosis is by histopathology with the identification of the pathogen in its diverse stages, rather than the stromal and cellular responses of the host. ${ }^{2,5}$ Surgery by hot or cold snare technique is the treatment of choice and endoscopic removal of naso-oropharyngeal polyps is also practiced (Arun et al 2009). Most patients with localized disease respond to treatment with Tablet Dapsone (which appears to arrest the maturation of the sporangia and to promote fibrosis in the stroma) following surgical excision. ${ }^{1-3}$ Tablet Dapsone is recommended up to 1-2 years to avoid recurrence \& dessimination. ${ }^{2,3}$ Local surgical excision of Nasal Rhinosporidiosis performed (Fauzia et al 2015). Total excision of the mass, preferably by electro-cautery, is recommended. ${ }^{1,3,5,6}$ However Laser provides adequate cauterization with good haemostasis thereby reducing the chances of complications and recurrence. ${ }^{7}$ Hence in our case $\mathrm{CO}_{2}$ laser was used over the surface attachments at nasal septum upto posterior choana and nasopharynx roof to minimise recurrence .Laser can be used as an adjunct during surgery and all measures to be taken so that the disease is removed completely and its attachments are cauterized or laserised after disease bulk removal. ${ }^{3}$ Early detection and surgical management will reduce significantly associated morbidity and mortality. ${ }^{3,5}$

\section{Conclusion}

Rhinosporidiosis and its causative pathogen R. seeberi have been known for over a hundred years. ${ }^{1,8,9}$ The organism is aquatic in nature and difficult to isolate in culture. Prevention will be the best option to be safe from this organism as the disease takes a chronic course which makes diagnosis difficult. Hence swimmers and persons who are frequent visitors to water bodies should have safety precautions as this organism get transferred through cut wounds. In view of the molecular characterization of the aetiological agent, further research to discover effective treatment options is needed. We found use of Tablet Dapsone is effective and safe medical treatment to arrest further growth preoperatively and to reduce recurrence postoperatively if used up to one year duration. Laser is relatively a new tool and it can be used safely as an adjunct especially at nasal septum, nasal floor, posterior choana and nasopharynx region.

\section{Acknowledgments}

None.

\section{Conflict of interest}

The author declares that there is no conflict of interests regarding the publication of this paper.

\section{References}

1. Jeshina Janardhana. Elusive treatment for human Rhinosporidiosis. Int $J$ Infect Dis. 2016;48:3-4.

2. de Silva NR, Huegel H, Atapattu DN, et al, Cell-mediated immune responses in human Rhinosporidiosis. Mycopathologia 2001;152(2):59 68

3. Tiwari. Rhinosporidiosis: A Riddled Disease of Man and Animals. Advances in Animal and Veterinary Sciences. 2015;3(2):55.

4. Aroor R, Gowda MM, Bhat VS, et al. Novel Approach to Rhinosporidiosis. Otorhinolaryngology Clinics: An International Journal. 2015.

5. Kameswaran S, Lakshmanan M. Rhinosporidiosis. In: ENT disorders in a tropical environment. Kameswaran S, Kameswaran M, editors. (MERF Publications, Chennai), 1999; p. 19-34. 
6. Das S, Kashyap B, Barua M, et al. Nasal Rhinosporidiosis in humans: new interpretations and a review of the literature of this enigmatic disease. Med Mycol. 2011;49(3):311-315.

7. Vinod M. K et al, Rare case of Rhinosporidiosis: a case report from Jammu. International Journal of Otorhinolaryngology and Head and Neck Surgery. 2017;3(1):148-150.
8. Jain S. Aetiology and Incidence of Rhinosporidiosis. Indian Journal of Otorhinology. 1967;19(1):1-21.

9. Fauzia. Nasal Rhinosporidiosis: A Case Study. Journal of Medical Microbiology \& Diagnosi., 2015;4(2). 